Sección uno: Ensayo

Radiografía de la innovación educativa en el Siglo XXI

\title{
Input musical y fluidez lectora en el desarrollo de la expresión escrita en estudiantes adultos de español como L2 ${ }^{1}$
}

Musical input and reading fluency in the development of written skills in adult L2 Spanish learners

\author{
Caterina Martínez Montiel \\ Universidad Internacional de \\ Valencia \\ caterinamm1414@gmail.com \\ Francisco Herrero Machancoses \\ Universidad Jaume I \\ herrerof@uji.es \\ José Manuel Foncubierta Muriel \\ Universidad de Huelva / KU \\ Leuven \\ jose.foncubierta@dfilo.uhu.es
}

\section{Resumen}

La música comparte propiedades con el lenguaje. A lo largo de los años se ha estudiado el vínculo entre ambas y su impacto en el aprendizaje de una L2. En el presente artículo nos centramos en estudiar el desarrollo de la expresión escrita y su relación con dos variables: la fluidez lectora y el input musical. Este pequeño estudio experimental se sitúa en el marco de la investigación en acción. La muestra estuvo compuesta por un total de 13 estudiantes de español de nivel B1, que se dividieron en dos grupos: uno de ellos recibió estímulo musical y el otro no. Asimismo, a ambos grupos se les facilitó un test de expresión escrita, basado en una actividad de Mario Rinvolucri, y un test de fluidez lectora oral. El estudio correlacional indica que es posible que haya una influencia positiva en el uso de la música como input añadido al texto escrito.

\footnotetext{
${ }^{1}$ Recibido: 11/01/2020 Evaluado: 10/02/2020 Aceptado: 27/02/2020
} 
Palabras clave: Input musical, expresión escrita, fluidez lectora, L2, contexto emocional

\begin{abstract}
Music shares characteristics with language. The link between both and their impact on second language learning has been studied over the years. In this paper we focus on studying the development of writing and its relation with two variables: reading fluency and musical input. This experimental study fits within an action-research framework. The sample used on this project consisted of 13 students of Spanish with a B1 level of language proficiency. The participants were divided into two groups, one of them received musical stimulation while the other did not. Additionally, both groups were given a writing test, based on an activity created by Mario Rinvolucri, along with an oral reading fluency test. The correlational study points out that there may be a positive effect on the use of music as input added to the written text.
\end{abstract}

Keywords: Musical input, writing, reading fluency, L2, emotional context

\title{
Introducción
}

Escribir no es una tarea fácil, requiere tiempo y concentración. La expresión escrita es la más cognitiva de todas las destrezas lingüísticas (Latif, 2013, p. 101) y el proceso es, además, mucho más complejo cuando se escribe en una segunda lengua (L2). Entre las dificultades a las que se enfrentan los estudiantes a la hora de escribir libremente un texto se encuentra el número de palabras que son capaces de recuperar durante el acto de composición en una tarea temporizada, su fluidez escrita. Esos síntomas de falta de ideas pueden fomentar la impresión de cierta pobreza léxica, a pesar de que disponen de una buena competencia gramatical y un buen nivel de comprensión lectora.

La motivación es clave para el aprendizaje de un idioma en edad adulta. Los objetivos de aprendizaje de las habilidades escritas suelen depender de la importancia que los estudiantes atribuyen a la escritura en la L2, de sus necesidades, su interés y las actitudes que mantienen hacia lo escrito (Kormos, 2012, p.394). En este contexto, el hábito lector como fuente de la experiencia con lo escrito y la música como fuente de motivación para el alumnado en el proceso de aprendizaje pueden ser variables interesantes para fomentar el desarrollo de la escritura fluida.

Stephen Krashen $(1984,1985)$ señaló la importancia de la lectura extensiva en el proceso de adquisición de la fluidez lectora, de modo que aquellos estudiantes que disponen de una buena actitud y disposición hacia la lectura, suelen obtener resultados más favorables en fluidez, en comprensión, léxico y, por ende, en su expresión escrita y conocimiento gramatical frente a aquellos alumnos sin hábito lector o problemas de fluidez lectora. En este sentido, la literatura dedicada al estudio de la lectura y su impacto en el desarrollo de la expresión escrita evidencia que ambas destrezas se hallan en una relación de dependencia, es decir, "el proceso de desarrollo de la escritura forma parte del proceso de adquisición del lenguaje y, en consecuencia, de la lectura” (Romero, 2004, p.4). 
La relación entre la adquisición de la fluidez lectora y su influencia directa en el desarrollo de las capacidades cognitivas de los sujetos fue descrita también por Stanovich (1986, Cunningham \& Stanovich, 1997) como el denominado "Efecto Mateo", el cual indica que quien tiene hábito lector no solo desarrolla su habilidad lectora, sino también otros elementos de la inteligencia verbal.

Aunque en el caso de la lectura se asume el valor de la Hipótesis del Input Comprensible y también que la tipología de los textos empleados determina el estilo de la escritura facilitando la adquisición, de manera inconsciente, de la competencia para escribir (Krashen 1985), debemos añadir que no existe una correlación directa entre la cantidad de lectura y la calidad de la escritura. Un buen estilo de escritura se adquiere tras una continua lectura mientras se escribe. Por tanto, la propia expresión escrita se debe ejercitar.

En el presente estudio se presenta, además, la hipótesis de que una adecuada introducción de la música instrumental como estímulo para el desarrollo de la libre expresión escrita en conjunción con la fluidez lectora puede contribuir a que los alumnos de español como L2 sean capaces de desarrollar una escritura más fluida y de calidad.

\section{Marco teórico}

En los últimos años el efecto de la música en el aprendizaje de una L2 ha venido recibiendo cada vez más atención. Schellenberg and Weiss (2013) sostienen que hay cuatro aspectos de la musicalidad que pueden afectar las habilidades cognitivas: la aptitud musical, la formación musical, escuchar música antes de realizar una tarea cognitiva y escuchar música mientras se realiza una tarea cognitiva. Los dos primeros aspectos han sido ampliamente abordados por la investigación, disponiéndose en la actualidad de suficientes evidencias que demuestran que música y lenguaje no son componentes separados y que las partes del cerebro que procesan la información musical se solapan con áreas neuronales responsables del procesamiento del lenguaje verbal (Patel, 2008; Slevc, 2012, Besson, Barbaroux \& Dittinger, 2017). Tanto en los estudios centrados en una población de no-músicos con habilidades musicales innatas (musical aptitude) como aquellos focalizados en sujetos con formación musical (musical training) han hallado correlaciones positivas entre las habilidades de percepción musical y componentes del lenguaje verbal como la conciencia fonológica o la sintaxis. En el contexto del aprendizaje de una segunda lengua, la obra seminal de Slevc \& Miyake (2006) aportó evidencias de correlación positiva entre la aptitud musical en personas adultas no formadas musicalmente y su capacidad de comprender y pronunciar mejor los sonidos de una L2.

Sin embargo, más allá de la influencia de las habilidades musicales en el desarrollo cognitivo de los componentes del lenguaje verbal, otra parte de la investigación se ha ocupado también de estudiar la influencia de la musicalidad en el desarrollo de destrezas lingüísticas como la comprensión lectora y la expresión escrita. El metaanálisis de Gordon, Fehd \& McCandliss (2015) y la revisión sistemática de Zeromskaite (2014) muestran el interés que ha recibido el estudio de las relaciones entre las destrezas de percepción musical y las habilidades lectoras tanto en lengua materna como en una segunda lengua. Sin embargo, continúa siendo objeto de debate si la transferencia de habilidades musicales al área del desarrollo de habilidades del lenguaje se debe a cuestiones de aptitud musical (Schellenberg, 2015) o si está motivada 
por los efectos de un entrenamiento o formación musical (Besson, Barbaroux \& Dittinger, 2017).

En el ámbito de la didáctica de segundas lenguas, Failoni (1993) señala que las actividades musicales pueden llegar a ser una herramienta más del aula, capaces de mejorar las habilidades comunicativas y el conocimiento cultural de los estudiantes, especialmente en los primeros niveles de aprendizaje. De hecho, las actividades musicales apoyadas en canciones han estado muy presentes en las clases de lenguas extranjeras. La música instrumental, por el contrario, no suele aparecer como recurso disponible para acompañar determinadas tareas en los libros de texto, a pesar de que su uso podría estimular la memoria verbal y contribuir en el recuerdo de estructuras gramaticales, vocabulario, elementos que se pueden utilizar para desarrollar las cuatro destrezas lingüísticas, etc. Tal y como proponía, en parte, el método de enseñanza de Lozanov en los años 70 denominado Sugestopedia, la música puede sugestionar positivamente al alumnado en su aprendizaje de una L2 (Foncubierta \& Grant, 2016).

Para Failoni (1993), la música se puede incorporar en el desarrollo de cualquier destreza en cualquier idioma y adaptarse a todos los niveles y edades. Sin embargo, la investigación centrada en el efecto del input musical sobre el desarrollo de tareas de expresión escrita continúa siendo escasa y sus resultados controvertidos. De un lado, estudios pioneros como los de Jensen (1931) así como investigaciones más próximas en el tiempo, como la de Ransdell and Gilroy (2001), han coincidido en señalar el efecto distractor de la introducción de input musical sobre la realización de tareas cognitivas como copiar un texto mientras se escucha música o escribir un ensayo. Jiang \& Sengupta (2011) concluyeron que la música producía una disminución en la capacidad productiva. Estos estudios refrendan la Hipótesis de la capacidad cognitiva, la cual establece que el procesamiento de la música puede interferir en el proceso cognitivo de la tarea lingüística (Schellenberg \& Weiss, 2013). De otra parte, Rauscher, Shaw, and Ky (1993) estudiaron el denominado "Efecto Mozart”. En su estudio se afirma que escuchar música de este compositor eleva el coeficiente intelectual. Sin embargo, Huckabee (2013) sostiene que aún no es posible concluir la validez de tal efecto, pues el estudio se ha replicado más de una vez y los resultados han sido contradictorios. Debe realizarse más investigación para determinar si la música puede afectar o no la capacidad cognitiva de los estudiantes que aprenden una segunda lengua. Estudios como los de Ho (2003) evidencian, al menos, que la música afecta positivamente la memoria verbal de los niños, lo cual está directamente conectado con la fluidez. En un estudio de Gibson, Folley, and Park’s (2009) se halló que los individuos que tenían formación musical fueron más creativos que los no-músicos y que la creatividad mantenía una relación positiva con la habilidad verbal. Lo cual añadíría un nuevo componente al hecho de evaluar la expresión escrita ya que se diferencia entre la fluidez en la escritura (número de palabras escritas en un tiempo limitado) y la calidad de lo escrito (riqueza expresiva). Kämpfe, Sedlmeier y Renkewitz (2011) determinaron que, aunque la música tenía algún efecto negativo en la memoria, tuvo un impacto significativo en las respuestas emocionales de los estudiantes. Asimismo, percibieron un efecto distinto en los procesos cognitivos dependiendo del ritmo y del estilo musical. Huckabee (2013) sostiene que el uso de input musical en su investigación (canciones de jazz) sí que propició un efecto positivo en algunos estudiantes, sin embargo, señala que la música de fondo puede ser positiva en algunos estudiantes y en otros ser un elemento de distracción. Kariuki \& Honeycutt (1998) partieron de la suposición 
de que a los estudiantes no les gusta escribir, mientras que sí disfrutan escuchando música. Su investigación demostró que la música es capaz de propiciar un ambiente que estimula su expresión creativa, la motivación, así como el desarrollo de la lectura y la escritura. Este último estudio, sitúa la introducción de input musical como un estímulo que ayuda a ejercitar la creatividad y la imaginación, además de amenizar el proceso cognitivo de la escritura.

La fluidez lectora entendida como "la destreza de leer con rapidez, precisión y con la expresión, entonación y pronunciación adecuada” tiene una evidente conexión con la comprensión de textos (Gómez, 2016, p.3). Los estudiantes con mayor nivel de fluidez lectora en una L2 tienen, en efecto, un nivel de comprensión lectora superior en la lengua meta. Un buen nivel de fluidez lectora proporciona más recursos para el desarrollo de la expresión escrita (Romero, 2004). Entre los tipos de prueba mayormente empleados para la medición de la fluidez lectora oral, se encuentra la tasa lectora (Lems, 2003). Un tipo de prueba consistente en la medición del número de palabras leídas correctamente en un minuto.

Este estudio se dirige a explorar la influencia del estímulo musical en el desarrollo de la expresión escrita, tomando también como referencia el impacto de otra variable como la fluidez lectora oral. Para ello, hemos establecido las siguientes preguntas de investigación:

1. ¿Existen diferencias significativas en las variables de expresión escrita según estén los sujetos en el grupo musical o no?

2. Si la respuesta es que sí, ¿cuál es la variable de la expresión escrita sobre la que más influye (número de palabras, número de adjetivos, complejidad sintáctica, número de conectores)?

3. ¿El nivel de fluidez lectora oral mantiene alguna relación con el desarrollo de la expresión escrita (número de palabras escritas)?

\section{Metodología}

Para llevar a cabo este pequeño estudio experimental, nos situamos en el marco de la investigación en acción, con el fin de obtener datos a partir de una situación real en el aula, en el que tuvieran que trabajar con actividades cotidianas.

\section{Participantes}

En este estudio participaron 13 alumnos de español de entre 20 y 30 años, matriculados en un curso de nivel B1 en la escuela Delengua, en Granada. El 31\% hombres y el 69\% mujeres. De estos 13 alumnos, 8 (61.5\%) eran de países nativos en habla diferente al inglés, y 5 (38.5\%) eran de países nativos en habla inglesa.

Los estudiantes fueron divididos aleatoriamente en dos grupos. Por un lado, un grupo de control (grupo A), sin ningún tipo de estímulo musical. Y, por otro lado, un grupo experimental (grupo B) que recibió input musical. 


\section{Instrumentos}

\subsection{Test de expresión escrita}

En primer lugar se administró un test para la redacción libre. Con el texto narrativo titulado "El Unicornio", los estudiantes tenían que leer una historia y completarla en un tiempo de 10 minutos. A lo largo del test debían describir a los distintos personajes, narrar las situaciones expuestas e inventar un final para la historia. Los estudiantes correspondientes al grupo A (grupo de control) realizaron el test sin ningún tipo de estímulo musical, mientras que los estudiantes del grupo B (grupo experimental) recibieron input musical basado en distintas composiciones musicales relacionadas con los diferentes personajes o situaciones de la historia para comprobar si, efectivamente, el estímulo musical tenía o no relación con el desarrollo de la escritura. La expresión escrita fue considerada a partir de subcomponentes como el número de palabras escritas, complejidad sintáctica (número de oraciones subordinadas), número de conectores discursivos y riqueza de adjetivos.

\subsection{Test de fluidez lectora oral}

El segundo test consistió en la lectura de un texto narrativo de 250 palabras. Todos los estudiantes debían leer en voz alta durante un minuto a una velocidad normal, pero atendiendo al significado del texto. A continuación, debían hacer un pequeño resumen. Para la recogida de datos se empleó la grabadora de un móvil iPhone 7 Plus y la fluidez lectora oral se computó de acuerdo con el número de palabras correctamente leídas en un minuto.

\section{Análisis estadístico}

Se llevó a cabo un análisis no paramétrico de comparación de grupos mediante la U de MannWhitney, dado el escaso tamaño muestral, con el fin de determinar si existen diferencias significativas entre el grupo Musical - No Musical. Para determinar la posible relación entre el nivel de fluidez lectora oral con el desarrollo de la expresión escrita, se realizó un estudio correlacional mediante la prueba Rho $(\rho)$ de Spearman. En ambas pruebas se ha implementado un análisis boostrap de Montecarlo basado en 10000 muestras de los datos, a un nivel de significación del 99\%, con el fin de suplir el escaso tamaño muestral.

\section{Resultados}

Tabla 1. Estadísticos de contraste no paramétricos

\begin{tabular}{lccccccc}
\hline & $\mathrm{U}_{\mathrm{M}-\mathrm{W}}$ & $\mathrm{P}$ & $\mathrm{p}$ (exact) & \multicolumn{2}{c}{ Monte Carlo } \\
& & & & $\mathrm{p}$ & $\mathrm{IC} 95 \%$ \\
\hline $\mathrm{N}^{\mathrm{o}}$ PALABRAS ESCRITAS & 10 & .116 & $.138_{\mathrm{a}}$ & .070 & .065 & .075 \\
\hline
\end{tabular}




\begin{tabular}{|c|c|c|c|c|c|c|}
\hline \multirow[b]{3}{*}{ COMPLEJIDAD SINTÁCTICA } & \multirow{3}{*}{$\begin{array}{c}\mathrm{U}_{\mathrm{M}-\mathrm{W}} \\
9.5\end{array}$} & \multirow{3}{*}{$\begin{array}{c}\mathrm{P} \\
\\
.095\end{array}$} & \multirow{3}{*}{$\begin{array}{c}\mathrm{p}(\text { exact }) \\
.101_{\mathrm{a}}\end{array}$} & \multicolumn{3}{|c|}{ Monte Carlo } \\
\hline & & & & \multirow{2}{*}{$\frac{\mathrm{p}}{.060}$} & \multicolumn{2}{|c|}{ IC 95\% } \\
\hline & & & & & .055 & .065 \\
\hline $\mathrm{N}^{\mathrm{a}}$ CONECTORES & 9.5 & .093 & $.101_{\mathrm{a}}$ & .058 & .054 & .063 \\
\hline RIQUEZA DE ADJETIVOS & 8.5 & .069 & $.073_{\mathrm{a}}$ & $.038 *$ & .034 & .042 \\
\hline FLUIDEZ LECTORA ORAL & 14.5 & .352 & $.366_{a}$ & .200 & .192 & .208 \\
\hline
\end{tabular}

Variable de agrupación: Música

a. No corregido para empates.

Tabla 2. Matriz de correlaciones Rho ( $\rho$ ) de Spearman.

\begin{tabular}{|c|c|c|c|c|c|c|}
\hline & & FLO & $\mathrm{N}^{\mathrm{o}} \mathrm{P}$ & CS & $\mathrm{N}^{\mathrm{a}} \mathrm{C}$ & RA \\
\hline \multirow{3}{*}{ FLUIDEZ LECTORA ORAL (FLO) } & $\mathrm{P}$ & 1,000 & & & & \\
\hline & $\mathrm{P}$ & & & & & \\
\hline & $\mathrm{P}$ & ,361 & 1,000 & & & \\
\hline \multirow[t]{2}{*}{$\mathrm{N}^{o}$ PALABRAS ESCRITAS $\left(\mathrm{N}^{\circ} \mathrm{P}\right)$} & $\mathrm{P}$ & ,226 & . & & & \\
\hline & $\mathrm{P}$ & ,031 & ,430 & 1,000 & & \\
\hline \multirow[t]{2}{*}{ COMPLEJIDAD SINTÁCTICA (CS) } & $\mathrm{P}$ & 921 & ,143 & . & & \\
\hline & $\mathrm{P}$ &,- 114 & ,578* & ,588* & 1,000 & \\
\hline Na CONECTORES $\left(\mathrm{N}^{\mathrm{o}} \mathrm{C}\right)$ & $\mathrm{P}$ & ,711 & 039 & ,034 & & \\
\hline
\end{tabular}




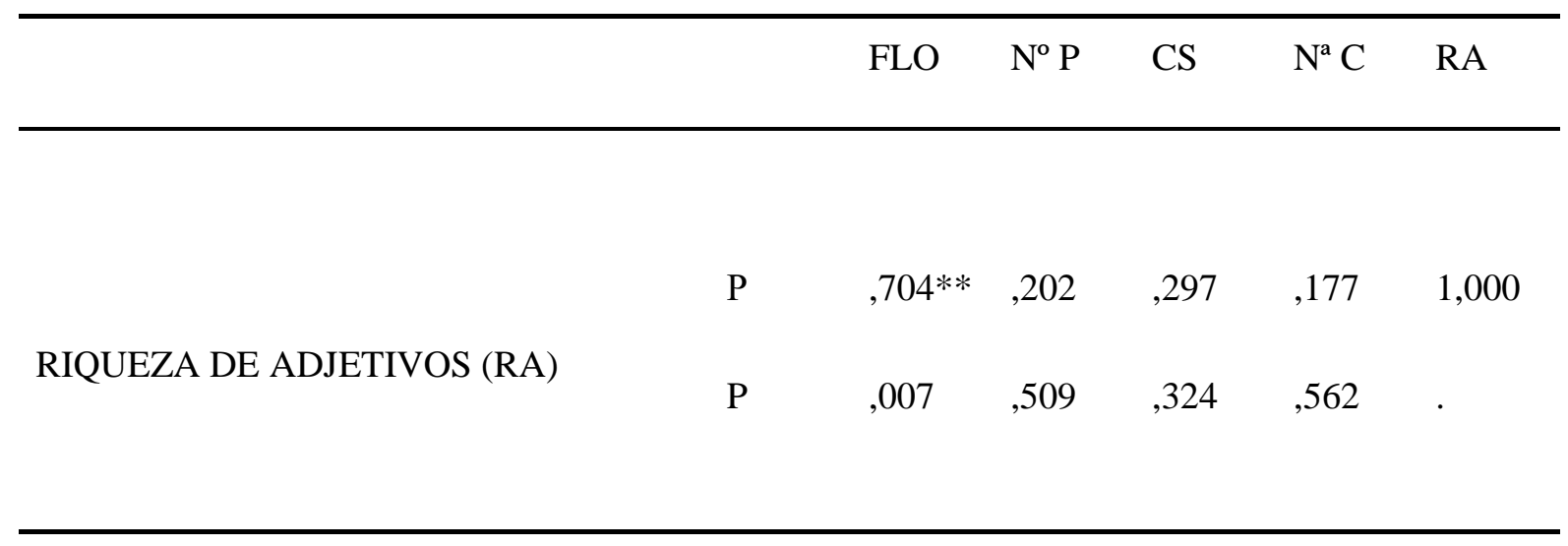

**. La correlación es significativa a un nivel de significación de 0.01 (2 colas).

*. La correlación es significativa a un nivel de significación de 0.05 (2 colas).

\section{Discusión}

PI 1. ¿Existen diferencias significativas en las variables de expresión escrita según estén los sujetos en el grupo musical o no?

Los resultados obtenidos indicaron una relación significativa entre la riqueza de adjetivos empleados por el grupo experimental (con música) y el grupo de control (sin música). Los datos aportados por Kariuki \& Honeycutt (1998) evidenciaron que el uso de la música influye positivamente, al motivar y estimular la imaginación de los individuos. Nuestro estudio coincide con esos resultados.

En estudios como el de Jensen (1931), centrados en si la música interfiere o no en la realización de otras tareas cognitivas (Hipótesis de la capacidad cognitiva, Schellenberg \& Weiss, 2013), se observó que la música era un distractor para los sujetos que tenían que mecanografiar un texto mientras escuchaban música. Asimismo, Ransdell and Gilroy (2001) observaron interferencia en la condición en la que unos sujetos tenían que escribir un ensayo mientras escuchaban música. En nuestra propuesta, sin embargo, el input musical no era un elemento exógeno a la tarea. El grupo experimental dispuso del estímulo musical para imaginar los personajes de la historia, de manera que la música era un lenguaje sonoro endógeno que enriquecía sensorialmente la actividad (Foncubierta \& Grant, 2016). Tal vez por este motivo nuestros resultados revelan una tendencia correlacional entre input musical y expresión escrita.

PI 2. Si la respuesta es que sí, ¿cuál es la variable de la expresión escrita sobre la que más influye (número de palabras, número de adjetivos, complejidad sintáctica, número de conectores)?

La variable de la expresión escrita sobre la que más influyó el input musical es la riqueza de adjetivos. La mayoría de los textos que debían componer los alumnos en un tiempo limitado eran de naturaleza descriptiva (describir un personaje masculino, describir un personaje femenino, etc.). Los alumnos que recibieron input musical describieron los personajes con 
una mayor riqueza léxica en lo concerniente a la diversidad y precisión de los adjetivos que aquellos que no recibieron ningún tipo de estímulo musical. El input musical estaría estimulando la posibilidad de generar imágenes mentales más nítidas de los personajes que protagonizan la historia. Por tanto, se evidencia que los estudiantes que recibieron estímulo musical durante la realización del test recuperaron y emplearon un mayor número de adjetivos. Aunque en este estudio no se consideraron variables como la formación musical o la experiencia musical, como en el estudio de Gibson, Folley, and Park's (2009), los resultados hallados remiten en cierto sentido a la posibilidad de que el input musical estimula los procesos creativos necesarios para la composición libre de textos escritos. Así, el grupo con estímulo musical estaría demostrando una mayor habilidad verbal en la condición experimental.

PI 3. ¿El nivel de fluidez lectora oral mantiene alguna relación con el desarrollo de la expresión escrita (número de palabras escritas)?

Los resultados indicaron que existe una correlación significativa entre la fluidez lectora y un aspecto de la expresión escrita, en este caso, la riqueza de adjetivos. Esto nos confirma que cuanto mayor sea la experiencia lectora del alumno, más disponibilidad léxica tendrá para el desarrollo de la expresión escrita, tal y como proponía Stanovich (1986) en su Hipótesis del Efecto Mateo, Krashen (1984, 1985) y Romero (2004).

\section{Conclusión}

Nuestro principal objetivo con este estudio preliminar era explorar si la fluidez lectora y el input musical mantienen alguna relación con el desarrollo de la expresión escrita.

De acuerdo con las investigaciones previas, hemos podido evidenciar la relación existente entre lectura y escritura, es decir, cuanto más fluidez lectora posee el alumno, mayor capacidad para expresarse por escrito, aunque la cantidad de lectura no determina la calidad de la escritura. Sin embargo, el estímulo musical puede enriquecer el proceso de desarrollo de la expresión escrita en términos de creatividad o calidad y riqueza de lo escrito. De manera extraña, a pesar de ser la música instrumental una herramienta didáctica de gran provecho y aportar numerosos beneficios tanto a nivel lingüístico como a nivel emocional, la revisión de los libros de texto para la enseñanza-aprendizaje del español, editados en España, muestra una ausencia de recursos e indicaciones para introducir el input musical en las aulas. En el ámbito de la investigación, los estudios en este área son aún escasos y los resultados contradictorios.

Este pequeño estudio experimental realizado en la escuela Delengua (Granada) nos indica que existe una tendencia positiva en la influencia de la música sobre la expresión escrita en alumnos de español como L2. Pero debemos señalar que por las limitaciones de este estudio, principalmente el tamaño muestral, conviene ser cautos. Aunque el trabajo ha aportado datos interesantes, se requiere de una investigación mayor. Un estudio que incluyera también la variable formación musical y un tamaño mayor de la muestra podría revelar datos aún más significativos para seguir estudiando la relación entre música y expresión escrita en segundas lenguas. 


\section{Referencias}

Besson, M., Barbaroux, M., \& Dittinger, E. (2017). Music in the brain: Music and language processing. In R. Ashley \& R. Timmers (Eds.), Routledge Companion to Music Cognition. New York, NY: Routledge / Taylor \& Francis.

Cunningham, A.E. \& Stanovich, K. E. (1997): Early Reading Acquisition and Its Relation to Reading Experience and Ability 10 Years Later. Developmental Psychology, 33 (6), 934-945.

Failoni, J. W. (1993) Music as Means to Enhance Cultural Awareness and Literacy in the Foreign Language Classroom. Mid-Atlantic Journal of Foreign Language Pedagogy, 7, 97-108.

Foncubierta, J.M \& Grant, M. (2016). “Awakening Senses for Language learning” en Fonseca-Mora, M.C. \& Grant, M. (Ed.). Melodies, Rhythm and Cognition in Foreign Language Learning. Cambridge Scholars Publishing.

Gibson, C., Folley, B. S., \& Park, S. (2009). Enhanced divergent thinking and creativity in musicians: A behavioral and near-infrared spectroscopy study. Brain and Cognition, 69, 162-169.

Gómez-Domínguez, M. (2016). Fluidez lectora oral en inglés como lengua extranjera. Tonos Digital, Revista de Estudios Filológicos, 31.

Gordon, R. L., Fehd, H. M., \& McCandliss, B. D. (2015). Does Music Training Enhance Literacy Skills? A Meta-Analysis. Frontiers in Psychology, 6. https://doi.org/10.3389/fpsyg.2015.01777

Ho, Y., et al. (2003). Music training improves verbal but not visual memory: Cross- sectional and longitudinal explorations in children. Neuropsychology, 17(3), 439- 450.

Jiang, X., \& Sengupta, A. K. (2011). Effect of music and induced metal load in word processing task. IEEE, 3261-3266

Huckabee, J. (2013). Improving Student Reading Fluency Scores through Music. A Capstone Submitted to the Gardner-Webb University School of Education in Partial Fulfillment of the Requirements for the Masters of Education.

Jensen, M. B. (1931). The influence of jazz and dirge music upon speed and accuracy of typing. Journal of Educational Psychology, 22(6), 458-462.

Kämpfe, J., Sedlmeier, P., \& Renkewitz, F. (2011). The impact of background music on adult listeners: A meta-analysis. Psychology of Music, 39(4), 424-448.

Lems, Kristen (2001): Using Music in the Adult ESL Classroom. ERIC Digest. 
Kariuki, P., \& Honeycutt, C. (1998). An investigation of the effects of music on two emotionally disturbed students' writing motivations and writing skills. (ERIC Document Reproduction Service No. ED427491).

Kormos, J. (2012). The role of individual differences in L2 writing. Journal of Second Language Writing, 21, 390-403.

Krashen, Stephen (1984) “Writing: research, theory and aplications”. Laredo Publishing Co: California.

Krashen, Stephen (1985) “The input hypothesis: Issues and implications”. Laredo Publishing Co: California.

Latif, M. M. (2013). What do we mean by writing fluency and how can it be validly measured? Applied Linguistics, 34(1), 99-105.

Lems, K. (2003). Adult ESL oral reading fluency and silent reading comprehension. Unpublished doctoral dissertation. Evanston, IL: National College of Education.

Patel, A. D. (2008). Music, language, and the brain. New York: Oxford University Press, Inc.

Ransdell, S. E., \& Gilroy, L. (2001). The effect of background music on word processed writing. Computer in Human Behavior, 17, 141-148.

Rauscher, F.H., Shaw, G.L., Ky,KN (1993). Music and spatial task performance. Nature, 365: 611

Romero Loaiza, F. (2004). Stephen D. Krashen: la lectura y su relación con la escritura. Repes: Revista Electrónica de Educación y Psicología, 1 (2).

Schellenberg, G. E., \& Weiss, M. W. (2013). Music and cognitive abilities. In D. Deutsch (Ed.), The psychology of music (3rd ed.) (pp. 499 - 550). London: Elsevier.

Schellenberg, E. G. (2015). Music training and speech perception: a gene-environment interaction. Annals of the New York Academy of Sciences, 1337(1), 170177. https://doi.org/10.1111/nyas.12627

Slevc, L. R., \& Miyake, A. (2006). Individual Differences in Second-Language Proficiency. Psychological Science,17(8), 675-681. ～https://doi.org/10.1111/j.14679280.2006.01765.x

Slevc, L. R. (2012). Language and music: sound, structure, and meaning. Wiley Interdisciplinary Reviews: Cognitive Science, 3(4), 483-492. https://doi.org/10.1002/wcs.1186 
Stanovich, K. (1986). Matthew Effects in Reading: Some Consequences of Individual Differences in the Acquisition of Literacy. Reading Research Quarterly, Vol. 21. (pp. 360-407)

Zeromskaite, I. (2014). The Potential Role of Music in Second Language Learning: A Review Article. Journal of European Psychology Students, 5(3), 78-88. https://doi.org/10.5334/jeps.ci 